\title{
Handheld, point-of-care laser speckle imaging
}

\author{
Ryan Farraro \\ Omid Fathi \\ Bernard Choi
}




\title{
Handheld, point-of-care laser speckle imaging
}

\author{
Ryan Farraro, ${ }^{\mathrm{a}, \mathrm{b}}$ Omid Fathi, ${ }^{\mathrm{c}}$ and Bernard Choi ${ }^{\mathrm{a}, \mathrm{b}, \mathrm{d}, \mathrm{e}, \mathrm{f}, *}$ \\ aUniversity of California-Irvine, Beckman Laser Institute and Medical Clinic, 1002 Health Sciences Road East, Irvine, California 92612, \\ United States \\ bUniversity of California-Irvine, Department of Biomedical Engineering, 3120 Natural Sciences II, Irvine, California 92697, United States \\ cUniversity of California-Irvine, Department of Pediatrics, 505 South Main Street, Irvine, Orange, California 92868, United States \\ dUniversity of California-Irvine, Department of Surgery, 333 City Boulevard West, Suite 1600, Orange, California 92868, United States \\ eUniversity of California-Irvine, Edwards Lifesciences Center for Advanced Cardiovascular Technology, 2400 Engineering Hall, Irvine, California \\ 92697, United States \\ ${ }^{f}$ CHOC Children's Hospital, 1201 West La Veta Avenue, Orange, California 92868, United States
}

\begin{abstract}
Laser speckle imaging (LSI) enables measurement of relative changes in blood flow in biological tissues. We postulate that a point-of-care form factor will lower barriers to routine clinical use of LSI. Here, we describe a first-generation handheld LSI device based on a tablet computer. The coefficient of variation of speckle contrast was $<2 \%$ after averaging imaging data collected over an acquisition period of $5.3 \mathrm{~s}$. With a single, experienced user, handheld motion artifacts had a negligible effect on data collection. With operation by multiple users, we did not identify any significant difference $(p>0.05)$ between the measured speckle contrast values using either a handheld or mounted configuration. In vivo data collected during occlusion experiments demonstrate that a handheld LSI is capable of both quantitative and qualitative assessment of changes in blood flow. Finally, as a practical application of handheld LSI, we collected data from a 53-day-old neonate with confirmed compromised blood flow in the hand. We readily identified with LSI a region of diminished blood flow in the thumb of the affected hand. Our data collectively suggest that handheld LSI is a promising technique to enable clinicians to obtain point-of-care measurements of blood flow. ๑ 2016 Society of Photo-Optical Instrumentation Engineers (SPIE) [DOI: 10.1117/1.JBO.21.9.094001]
\end{abstract}

Keywords: laser speckle contrast imaging; laser Doppler; neonatal intensive care unit; pediatrics; perfusion.

Paper 160273SSR received Apr. 25, 2016; accepted for publication Aug. 12, 2016; published online Aug. 31, 2016.

\section{Introduction}

Laser speckle imaging (LSI) is a noninvasive modality that enables measurement of relative changes in microvascular blood flow in biological tissues. ${ }^{1-4}$ Researchers have used LSI in a variety of pilot clinical tests to monitor local blood-flow changes. ${ }^{5-7}$ However, LSI is not used for routine hemodynamic monitoring in the clinic, mainly due to a lack of standardized instrumentation. We and other groups previously demonstrated that a mounted LSI device with sensitivity to blood-flow dynamics can be created with the use of inexpensive, consumer-grade electronics. ${ }^{8,9}$

Despite this advancement in ease of integration, there has been little work addressing ease of use. We postulate that a handheld, point-of-care form factor will lower barriers to routine clinical use of LSI for the diagnosis and monitoring of diseases associated with altered microvascular function. Since LSI is inherently sensitive to motion and hence corruption from motion artifact of the operator, a critical need exists to study the capabilities and limitations of handheld LSI and assess its feasibility in a clinical setting.

Herein, we describe a handheld LSI device with an untethered, compact form factor that we believe is well suited for bedside use. In contrast with previous related efforts, ${ }^{8,9}$ our design maintains use of scientific-grade cameras to achieve accurate measurements of regional blood flow. We present in vitro data collected by multiple users from tissue-simulating liquid phantoms and we present comparisons of those data to measurements from a mounted version of the same device. We also present in vivo data collected from human subjects using two occlusion protocols, which collectively demonstrate the ability of handheld LSI to enable both visual and quantitative characterization of regional blood flow. Lastly, as a proof-ofconcept measurement, we present clinical data obtained from a neonatal patient with decreased blood flow in the right hand. These data demonstrate that the device can successfully be deployed in a challenging clinical setting to identify regions of compromised blood flow.

\section{Materials and Methods}

\subsection{Use of Human Subjects}

The occlusion protocol and neonatal imaging measurements were approved by the Institutional Review Board of University of California, Irvine (ClinicalTrials.gov identifier NCT01484730 and NCT01483703, respectively).

\subsection{Handheld Laser Speckle Imaging Device}

Our LSI device (Fig. 1) consisted of a monochrome camera, laser pointer, and tablet computer. The monochrome camera was an 8-bit, 1.32 megapixel $(3.75-\mu \mathrm{m} \times 3.75-\mu \mathrm{m}$ pixel size $)$ CCD camera (CMLN-13S2M-CS, Point Grey, Richmond, Canada) equipped with a close-focus zoom lens (MLH-10x, Computar, CBC America Corp, New York). We attached the camera to the back of a Surface Pro 2 tablet (Microsoft, 


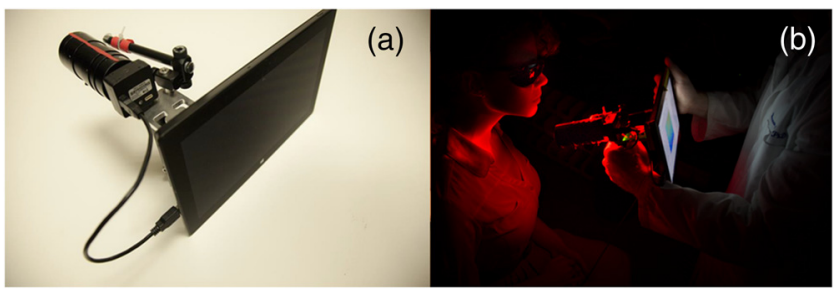

Fig. 1 (a) Tablet-based LSI system. (b) Representative example of system in use to collect LSI images from a human subject.

Redmond, Washington). For in vitro experiments, we attached a 650-nm center wavelength bandpass filter (Edmund Optics, Barrington, New Jersey) to the lens of the camera to mitigate the effects of room lighting on the collected images. For in vivo experiments, we performed the measurements with all room lights turned off. The pixel resolution of the images was $\sim 120$ pixels $/ \mathrm{mm}^{2}$.

For the excitation source, we used two lasers. For in vitro and clinical experiments, we used a 650 -nm laser pointer $(5 \mathrm{~mW}$, Five Star Inc., West Chester, Pennsylvania) covered with transparent tape to diffuse the beam. To obtain LSI images over a wide field of view during in vivo, laboratory measurements, we used a 50-mW, 637-nm laser (Dragon Lasers, ChangChun, China). The maximum optical penetration depth was $\sim 4 \mathrm{~mm}$ into skin, based on the wavelengths of the lasers used. $^{10}$

To facilitate image acquisition, we used custom-written software in MATLAB designed with a touch-sensitive graphical user interface. We attached both the camera and the laser to the back of the tablet via a custom-machined mount.

To ensure that the images were in focus each time the device was repositioned, we oriented the lens of the camera orthogonal to the tablet and the laser pointer at a slight ( $20 \mathrm{deg})$ angle with respect to the camera axis. With this alignment, the center of the laser spot moved laterally in the image as the user moved the device closer to or farther from the target. Using a solid reference object as a test target, we verified that, with the laser spot diameter $(25 \mathrm{~mm})$ and laser alignment angle, the depth of field $(149 \mathrm{~mm})$ of the device was sufficiently large to ensure that the collected raw speckle images remained in focus as long as the laser spot was visible within the field of view.

We converted all raw images into spatial maps of speckle contrast using a $7 \times 7$ pixel sliding window and the speckle contrast equation $K=\sigma /\langle I\rangle$, where $K$ is speckle contrast and $\sigma$ and $\langle I\rangle$ are the standard deviation and mean intensity, respectively, of 49 pixels within the window. ${ }^{11}$ The position of the sliding window was moved so that the window was centered on each pixel and the corresponding speckle contrast of that pixel calculated with the contrast equation. For each measurement, we collected a set of 250 images at a frame rate of $30 \mathrm{fps}$. We selected a $100 \times 100$ region of interest $(\mathrm{ROI})$ from each of the images and calculated the average speckle contrast from each of the 250 subimages. We then averaged the speckle contrast values to obtain a single average contrast value for each set of 250 subimages.

To facilitate comparison with previously published data, ${ }^{12}$ we converted speckle contrast values to maps of speckle flow index (SFI) using the simplified speckle imaging equation $\mathrm{SFI}=\left(2 T K^{2}\right)^{-1}$, where $T$ is exposure time and $K$ is speckle contrast. ${ }^{13}$ Previous work from our group suggests a linear response range between SFI and flow speed. ${ }^{14}$ SFI maps were used to visualize relative changes in blood flow.

\subsection{Tissue-Simulating Phantoms}

To simulate imaging of biological tissues, liquid Intralipid phantoms were synthesized for in vitro validation tests. Liquid phantoms were chosen, as dermal microvascular flow is well represented by Brownian motion, which is mimicked by a well-filled stationary liquid. ${ }^{15}$ The speckle contrast of a liquid phantom can be altered by changing the optical properties. LSI is sensitive to both changes in absorption and scattering, due to the effect of optical properties on modulating the pathlength of light. An increase in absorption leads to a suppression of longer pathlength photons, which in turns increases speckle contrast. ${ }^{16}$ Intralipid (1\%) was added to four 25.4-mm diameter dishes. With the addition of various quantities of India ink by weight, we generated four distinct phantoms with absorption coefficient values over a range $\left(0\right.$ to $\left.0.03 \mathrm{~mm}^{-1}\right)$ representative of biological tissues. ${ }^{17}$ The phantoms were used for all in vitro experiments.

\subsection{In Vitro Experiments}

We acquired images from each of the four phantoms with the device in either a handheld or a mounted configuration. To determine the effect of number of collected images on the variation in speckle contrast values calculated from each image, we acquired three sets of images of one of the tissue-simulating liquid phantoms. The coefficient of variation (COV) of speckle contrast was calculated using the equation $\mathrm{COV}=\sigma / \mu$, where $\sigma$ and $\mu$ are the standard deviation and mean, respectively, of the speckle contrast values for individual subsets of consecutive speckle contrast images. The number of images included in the COV calculation served as the independent variable.

To study the effect of motion artifacts associated with handheld operation of the LSI device, we imaged the tissue-simulating liquid phantoms at different camera exposure times. To compare the handheld data with the mounted data, we used logarithmic regression and performed a linear fit to the mounted data. We then calculated the sum of squares of the fit to the data and calculated the quality of fit for each phantom measurement.

We then investigated the repeatability of measurements across multiple users. Seven volunteers used the handheld device to image the same four liquid phantoms described above. The exposure time was set at $5 \mathrm{~ms}$ and the lens aperture at $f / 22$. Each user was instructed on how to use the device and subsequently collected three sets of images from each phantom. After each user acquired images from each of the four phantoms, we mounted the device in a fixed position and collected an identical number of images. We used a two-way ANOVA test to assess if the group mean for each phantom measurement differed significantly $(p<0.05)$ depending on $(1)$ the concentration of India ink, (2) the imaging configuration, and (3) any interaction between the concentration and configuration.

\subsection{In Vivo Experiments}

Two occlusion models were used to assess the qualitative and quantitative performance of the device to identify regions of altered blood flow. As a qualitative demonstration, we induced occlusion of the fingertip by tightly wrapping a rubber band around the knuckle of the finger of a volunteer. We illuminated 
the entire hand to obtain a wide-field blood-flow map in an attempt to identify a region of decreased blood flow to the occluded digit.

To demonstrate the ability of the device to provide a quantitative assessment of in vivo blood-flow dynamics, we performed a reactive hyperemia experiment ${ }^{8}$ on one human subject. We used a sphygmomanometer to apply a pressure of $180 \mathrm{mmHg}$ for $1 \mathrm{~min}$ to temporarily occlude the brachial artery. We held the LSI device to collect raw speckle images from the palm of the subject. We then released the applied pressure and continued to collect raw speckle images to visualize the known hyperemic response to the occlusion challenge. We then selected an ROI and calculated the average SFI value for each frame.

\subsection{Clinical Case Study: Neonatal Intensive Care Unit}

In an ongoing feasibility study, we used the handheld LSI device to collect data from a neonate, which developed a clot in the right forearm that was confirmed with Doppler ultrasound. Tissue downstream of the clot became hypoxic due to the blockage of flow. The peripheral vasculature in the thumb was most affected and led to irreversible tissue damage. Handheld LSI measurements were taken of the affected hand to determine if a perfusion deficit could be identified in the thumb.

\section{Results}

\subsection{In Vitro Experiments}

In general, the COV decreased with an increase in the number of frames that were averaged (Fig. 2). We postulate that the large fluctuations in the COV function when fewer frames are averaged are due to the distribution of the speckle contrast values approaching normality as the number of frames increased. The COV was less than $2 \%$ after averaging over 160 frames, which corresponds to an image acquisition period of $5.3 \mathrm{~s}$.

\subsubsection{Single, experienced user}

For each concentration of India ink, we observed a decrease in speckle contrast with an increase in exposure time (Fig. 3). The trend was similar between the data collected in a handheld and mounted configuration. Based on the $R^{2}$ coefficients $(0.72$ to 0.87) calculated from comparison of the handheld and mounted

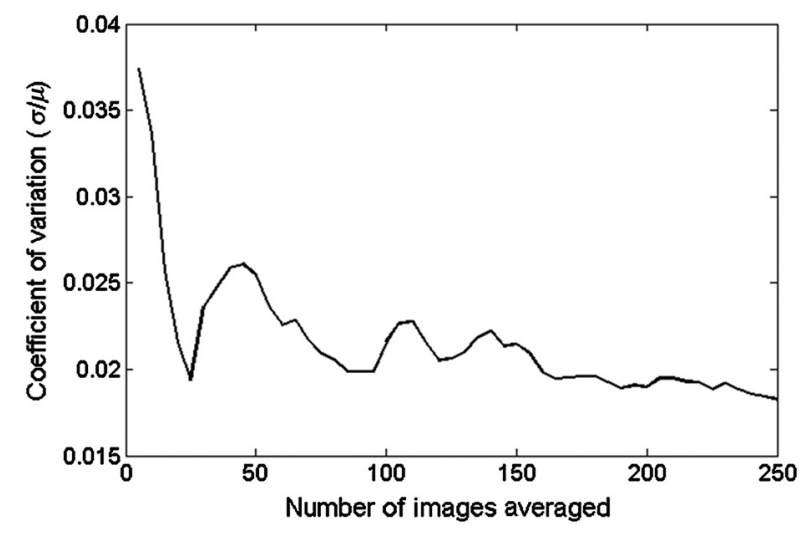

Fig. 2 COV calculated as a function of the number of frames averaged. The COV was calculated from each set of images $(n=3)$. The COV decreased below $2 \%$ with analysis of 160 frames collected over $5.3 \mathrm{~s}$.
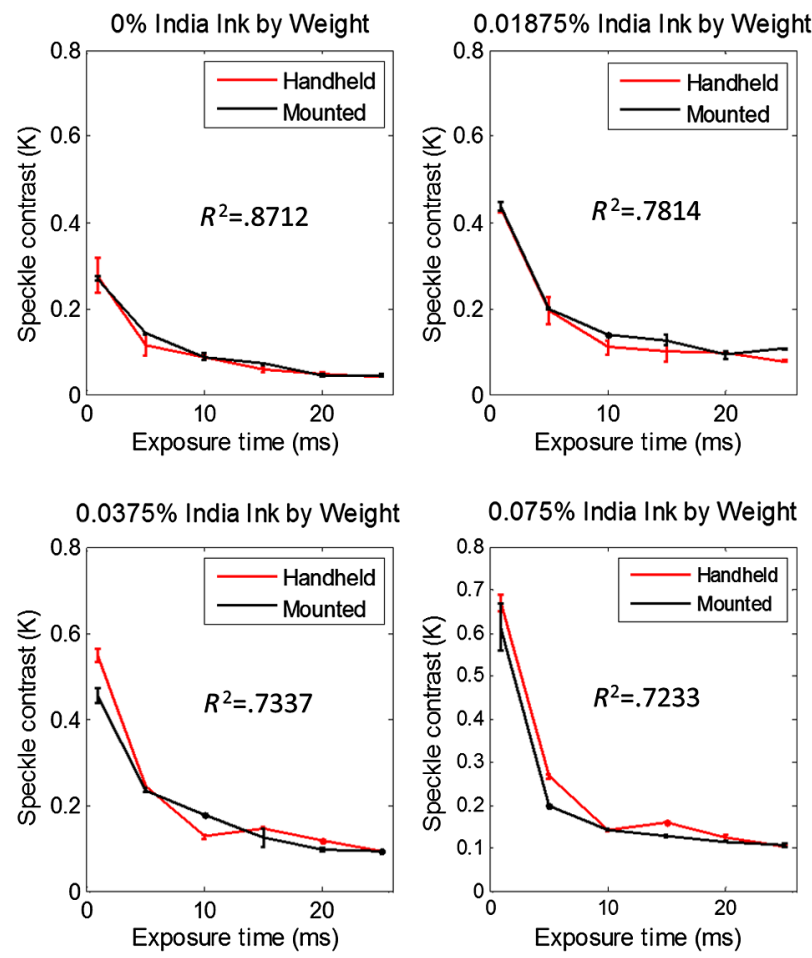

Fig. 3 Comparison of single-user handheld measurements of speckle contrast with those obtained using a mounted device. Over a range of absorption coefficients (varied with India ink fraction), speckle contrast values were similar with both configurations. Error bars represent the standard deviation of the mean contrast value $(n=4$ for each data point). The $R^{2}$ values represent the goodness of fit associated with comparison of the log of the handheld and mounted contrast values. Lines are used strictly as a visual aid for the reader and do not represent model-based fitting of the data.

data, we conclude that, with a single, experienced user, handheld motion artifacts had a negligible effect on the accuracy of the data collected with the device.

\subsubsection{Multiple users}

With operation by multiple users, we did not identify any significant difference $(p>0.05)$ between the measured speckle contrast values using either a handheld or mounted configuration (Fig. 4). We also did not identify any significant effect $(p>0.05)$ from the interactions between the configuration used during acquisition and the concentration of India ink. Finally, we identified a significant difference $(p<0.05)$ between the measured speckle contrast values of each concentration of India ink. Collectively, these data provide support of the ability of multiple operators to use handheld LSI to measure speckle contrast values that are similar to those measured with the conventional mounted configuration.

\subsection{In Vivo Experiments}

\subsubsection{Occlusion models}

With the fingertip occlusion model, we observed a decrease in SFI in the occluded fingertip compared with values in the surrounding regions of the hand (Fig. 5). The decrease in SFI coincides with the expected decrease in blood flow associated with the occlusion. These data support the ability of the handheld LSI 


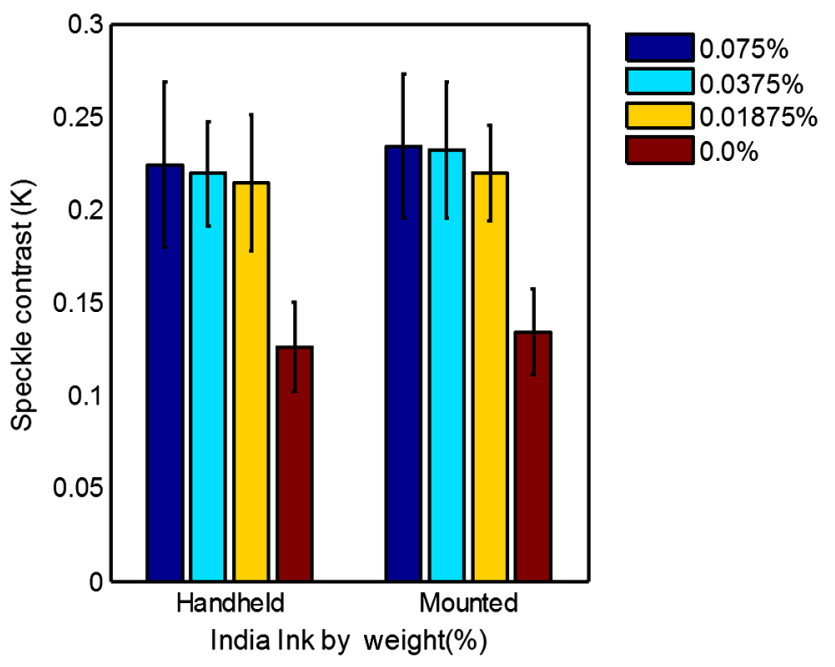

Fig. 4 Comparison of speckle contrast values from handheld measurements taken by multiple users $(n=7)$. Measurements collected with a mounted device are included for comparison. The mean contrast value was taken from each data set ( $n=3$ sets per user). The measured speckle contrast values were similar with both configurations. Error bars represent the standard deviation of the mean contrast value ( $n=21$ for each bar)
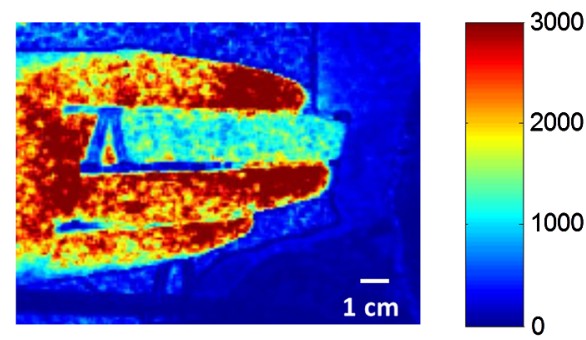

Fig. 5 SFI image of a hand during fingertip occlusion with a rubber band. We collected the raw speckle images using the LSI device in handheld mode. The occluded fingertip had a lower SFI value than the other fingertips.

device to qualitatively detect regions of compromised blood flow in a laboratory.

With the reactive hyperemia model, a decrease in SFI was observed, coinciding with the occlusion of the brachial artery from $t=20$ to $95 \mathrm{~s}$. The SFI values initially increased and returned to baseline after the occlusive pressure was released. These changes in SFI are in agreement with the expected trend of hyperemic blood flow following cuff release ${ }^{8}$ (Fig. 6). These data support the ability of handheld LSI to reliably image quantitative blood-flow dynamics from human subjects.

\subsubsection{Clinical case study: neonatal intensive care unit}

With handheld LSI of the hand of a neonate, we readily identified a region of compromised blood flow in the digit occluded due to a confirmed blood clot in the forearm (Fig. 7). These data suggest a potential role of handheld LSI in a challenging clinical environment such as the neonatal intensive care unit, to enable routine measurements of blood flow in regions of interest, potentially by nonexperts (i.e., clinical staff).

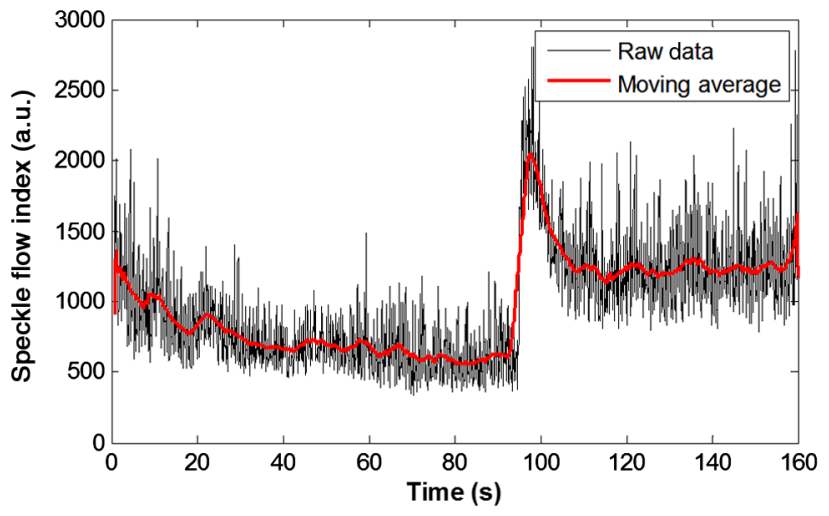

Fig. 6 Average SFI versus time measured from a human palm during brachial artery occlusion and release. A pressure of $180 \mathrm{mmHg}$ was applied at the 20-s mark and maintained until the 95-s mark. The applied pressure was then released, resulting in a hyperemic response. A moving average filter was applied to the average signal for clarity (in red).

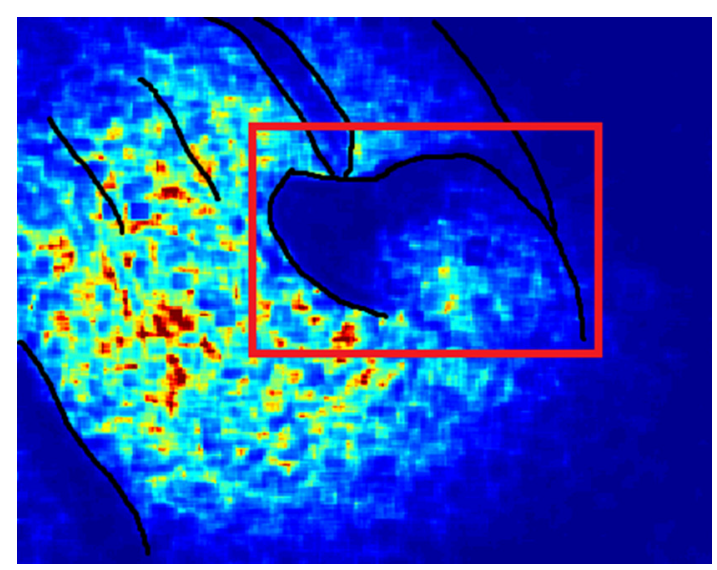

Fig. 7 Blood-flow map of the right hand of a neonate (53 days postgestation) in the neonatal intensive care unit at UC-Irvine Medical Clinic. The thumb (located inside of the red rectangle) of the subject had a clear reduction in blood flow, resulting from a clot in the right forearm. Red $=$ highest perfusion, dark blue = lowest perfusion . Note that an outline (in black) of the hand and fingers was superimposed for clarity.

\section{Discussion}

While microvascular blood flow has been studied using occlusion plethysmography ${ }^{18-20}$ and laser Doppler perfusion imaging, ${ }^{21-23}$ widespread clinical adoption of these techniques is impacted by several limitations, including long acquisition times and the need for contact measurements. We postulate that real-time, point-of-care, quantitative blood-flow measurements will facilitate routine measurements and help identify and monitor regions of compromised blood flow associated with diseases that affect the microcirculation, such as peripheral vascular disease and diabetes-related complications.

The data obtained with the device support our hypothesis that motion artifacts associated with handheld operation can be managed through image averaging. Our data demonstrate that (1) the $\mathrm{COV}$ associated with measurements can be reduced to less than $2 \%$ by averaging images collected over a 5.3-s acquisition period, (2) handheld measurements taken using biologically relevant exposure times ( 1 to $30 \mathrm{~ms}$ ) are similar to those taken 
with the device in a conventional mounted geometry, and (3) speckle contrast measurements taken by multiple novice users can be similar. To the best of our knowledge, these data represent the first demonstration of handheld LSI.

A robust handheld LSI device is expected to enable rapid assessment of blood flow in clinical environments in which both time and space are costly. With appropriate training, we envision that both clinicians and clinical staff can operate a handheld LSI device to collect measurements during routine monitoring of each patient, for a wide variety of clinical indications. As it is not feasible to have a trained technician on site at all times, we speculate that the handheld LSI device will be particularly useful to obtain measurements in time sensitive situations where perfusion could be monitored over a 24-h period such as in the intensive care unit or after surgery. Our pilot data collected using two occlusion protocols (fingertip occlusion and reactive hyperemia) demonstrate the ability of handheld LSI to image blood-flow dynamics in both a qualitative and quantitative manner.

One potential application of a handheld LSI device is in the field of neonatalogy and pediatrics. In highly sensitive and crowded areas such as the neonatal intensive care unit, compact, rapid instrumentation is required, especially for clinical trials in which both parental consent and acceptance by clinical staff is of paramount importance to data collection. Hence, we focused on development of our handheld LSI device around the use of a touchscreen PC, which is increasingly commonplace in the hospital setting. With use of a scientific-grade CCD camera, we ensure collection of raw speckle images that are comparable to those collected in traditional mounted laboratory configurations. As a proof of concept, we demonstrated that handheld LSI can identify a region of compromised perfusion in the thumb of a neonate, in agreement with Doppler ultrasound measurements. Further studies are warranted.

Stark et al. ${ }^{24-26}$ used laser Doppler flowmetry to study peripheral microvascular dysfunction in preterm infants associated with abnormal vascular resistance. Point-of-care LSI may enable similar measurements, with additional advantages of noncontact operation and wide-field imaging of a larger field of view. Furthermore, many neonatal diseases such as necrotizing enterocolitis (NEC), ${ }^{27}$ neonatal encephalopathy, ${ }^{28}$ polycythemia, ${ }^{29}$ and sepsis $^{30}$ are all associated with major ischemic events during the first days of postnatal life. Most of these diseases are asymptomatic in nature, and all of them require intervention to mediate blood flow. For example, the most common gastrointestinal disease affecting neonates is NEC, which is associated with major ischemic events in the mesenteric artery. Surgery is required to resect the gut once the tissue is identified as necrotic. ${ }^{27} \mathrm{~A}$ handheld device may allow a clinician or clinical staff to monitor the abdominal region to identify potential decreases in blood flow associated with the disease. LSI could also be used intraoperatively to aid clinicians in rapidly identifying unviable intestinal tissue during resection.

Our study has limitations. With the initial device design presented in this study, we rely solely on image averaging and did not explore alternate approaches to address motion artifact. Although we propose that an image acquisition period of $5 \mathrm{~s}$ is an acceptable tradeoff for the ease of use of a handheld, untethered LSI device, we acknowledge that this period can be reduced. Future work will involve study of strategies designed to reduce the number of images required to account for motion artifact, either of the subject or the operator. Research groups have proposed use of various strategies to correct for motion. ${ }^{31,32}$ Another approach is to use fiducial markers or accelerometers to identify images corrupted with motion artifact and reject those images from further analysis.

In conclusion, our in vitro and in vivo data suggest that handheld LSI is a promising technique to enable clinicians to obtain point-of-care measurements of blood flow. The results of the data suggest that LSI has the potential to provide useful hemodynamic information to clinicians that is not currently measured on a routine basis. Our in vivo data suggest that handheld LSI can detect physiological changes in blood flow similar to measurements taken with a mounted LSI device. Future work is warranted to refine or revise the device design and evaluate its performance in various clinics.

\section{Acknowledgments}

This research was funded in part by the Arnold and Mabel Beckman Foundation; the National Institutes of Health (R01 DE022831); the National Institutes of Health Laser Microbeam and Medical Program (P41 EB015890); the University of California, Irvine School of Medicine; and the American Society for Laser Medicine and Surgery. We would also like to thank Dr. Sean White, Dr. Bruce Yang, and Dr. Wesley Moy; and Dr. Ben Lertsakdadet, Dr. Caitlin Regan, and Dr. Christian Crouzet (all at University of California, Irvine) for their contributions to the in vitro experiments and providing insight on the development of handheld LSI.

\section{References}

1. A. F. Fercher and J. D. Briers, "Flow visualization by means of singleexposure speckle photography," Opt. Commun. 37, 326-330 (1981).

2. J. D. Briers, "Laser speckle contrast imaging for measuring blood flow," Opt. Appl. 37, 139-152 (2007).

3. D. A. Boas and A. K. Dunn, "Laser speckle contrast imaging in biomedical optics," J. Biomed. Opt. 15, 011109 (2010).

4. S. J. Kirkpatrick et al., "Quantitative temporal speckle contrast imaging for tissue mechanics," J. Opt. Soc. Am. A 24, 3728-3734 (2007).

5. A. B. Parthasarathy et al., "Laser speckle contrast imaging of cerebral blood flow in humans during neurosurgery: a pilot clinical study," J. Biomed. Opt. 15, 066030 (2010).

6. Y. C. Huang et al., "Blood flow dynamics after laser therapy of port wine stain birthmarks," Lasers Surg. Med. 41, 563-571 (2009).

7. N. Hecht et al., "Laser speckle imaging allows real-time intraoperative blood flow assessment during neurological procedures," J. Cereb. Blood Flow Metab. 33, 1000-1007 (2013).

8. O. Yang and B. Choi, "Laser speckle imaging using a consumer-grade color camera," Opt. Lett. 37, 3957-3959 (2012).

9. L. Richards et al., "Low-cost laser speckle imaging of blood flow using a webcam," Biomed. Opt. Express 4, 2269-2283 (2013).

10. L. Fodor, U. Yehuda, and M. Elman, "Light tissue interactions," in Aesthetic Applications of Intense Pulsed Light, pp. 11-20, SpringerVerlag, Berlin, Germany (2011).

11. J. W. Goodman, "Statistical properties of laser speckle pattern," Chapter 2 in Laser Speckle and Related Phenomena, J. C. Dainty, Ed., Vol. 9, pp. 9-75 (1975).

12. A. K. Dunn et al., "Dynamic imaging of cerebral blood flow using laser speckle," J. Cereb. Blood Flow Metab. 21, 195-201 (2001).

13. J. C. Ramirez-San-Juan et al., "Impact of velocity distribution assumption on simplified laser speckle imaging equation," Opt. Express 16, 3197-3203 (2008).

14. B. Choi et al., "Linear response range characterization and in vivo application of laser speckle imaging of blood flow dynamics," J. Biomed. Opt. 11, 041129 (2006).

15. Y. Shang, K. Gurley, and G. Yu, "Diffuse correlation spectroscopy for asessment of tissue blood flow in skeletal muscle: recent progress," Anat. Physiol.: Current Res. 3, 128 (2013). 
16. A. Mazhar et al., "Laser speckle imaging in the spatial frequency domain," Biomed. Opt. Express 2, 1553-1563 (2011).

17. P. Di Ninni, F. Martelli, and G. Zaccanti, "The use of India ink in tissuesimulating phantoms," Opt. Express 18, 26854-26865 (2010).

18. P. Y. Wu et al., "Peripheral blood flow in the neonate; 1 . Changes in total, skin, and muscle blood flow with gestational and postnatal age," Pediatr. Res. 14, 1374-1378 (1980).

19. G. Wallgren and J. Lind, "Observation of the newborn infants peripheral circulation and plasma expansion during moderate hypovolemia," Acta Paediatr. 56, 55-68 (1967).

20. M. J. Stark, V. L. Clifton, and I. M. Wright, "Microvascular flow, clinical illness severity, and cardiovascular function in the preterm infant," Arch. Dis. Child. - Fetal Neonat. Ed. 93, F271-F274 (2008).

21. R. M. Dyson et al., "Early microvascular changes in the preterm neonate: a comparative study of the human and guinea pig," Physiol. Rep. 2, e12145 (2014)

22. A. C. Yao et al., "Peripheral circulatory response to feeding in the newborn infant," Pediatrics 47, 378-383 (1971).

23. J. W. Lott, "Skin blood flow responses to thermal stimuli in healthy term newborns," Newborn Infant Nurs. Rev. 3, 179-183 (2003).

24. M. J. Stark, V. L. Clifton, and I. M. Wright, "Neonates born to mothers with preeclampsia exhibit sex-specific alterations in microvascular function," Pediatr. Res. 65, 291-295 (2009).

25. M. J. Stark, V. L. Clifton, and I. M. Wright, "Carbon monoxide is a significant mediator of cardiovascular status following preterm birth," Pediatrics 124, 277-284 (2009).

26. M. J. Stark et al., "The influence of sex and antenatal betamethasone exposure on vasoconstrictors and the preterm microvasculature," J. Matern.-Fetal Neonat. Med. 24, 1215-1220 (2011).

27. M. J. Bell et al., "Neonatal necrotizing enterocolitis. Therapeutic decisions based upon clinical staging," Ann. Surg. 187, 1-7 (1978).
28. G. Hankins and M. Speer, "Defining the pathogenesis and pathophysiology of neonatal encephalopathy and cerebral palsy," Obstet. Gynecol. 102, 628-636 (2003).

29. R. S. Ramamurthy and Y. W. Brans, "Neonatal polycythemia: I. Criteria for diagnosis and treatment," Pediatrics 68, 168-174 (1981).

30. S. A. Quazi and B. J. Stoll, "Neonatal sepsis: a major global public health challenge," Pediatr. Infect. Dis. J. 28, S1-S2 (2009).

31. L. M. Richards et al., "Intraoperative laser speckle contrast imaging with retrospective motion correction for quantitative assessment of cerebral blood flow," Neurophoton 1, 015006 (2014).

32. L. Omarjee et al., "Optimisation of movement detection and artifact removal during laser speckle contrast imaging," Microvasc. Res. 97, 75-80 (2015).

Ryan Farraro received his BS and MS degrees in biomedical engineering from University of California, Irvine. He currently is a product development engineer for stability biologics.

Omid Fathi received his MD from the Ohio State University College of Medicine. After completing residency and fellowship training at University of California, Irvine, he joined the physician team at Nationwide Children's Hospital in Columbus, $\mathrm{OH}$.

Bernard Choi is an associate professor of biomedical engineering and surgery at $\mathrm{UCl}$, with appointments at Beckman Laser Institute and Medical Clinic, Edwards Lifesciences Center for Advanced Cardiovascular Technology, and CHOC Children's Hospital. His current research is in the field of vascular biophotonics, with current emphases in optical hemodynamic monitoring, microvascular dynamics of tissue in normal and proliferative states, and optical clearing. 